\title{
XXVI. Some observations on the theory and application of electricity
}

\section{F.C. Henrici}

To cite this article: F.C. Henrici (1845) XXVI. Some observations on the theory and application of electricity , Philosophical Magazine Series 3, 27:179, 164-171, DOI: 10.1080/14786444508645251

To link to this article: http://dx.doi.org/10.1080/14786444508645251

曲 Published online: 30 Apr 2009.

Submit your article to this journal $₫$

Q View related articles $₫$ 
Minerals.

Sodalite, Vesuvius.

Variety of hypersthene, occurring in the diorite on the Lahn, Nassau.

Lepidolite, locality unknown.

Obsidian, from Lipari.

Mica, Spessart, Bavaria.

Granite, Odenwald, Germany.

Chlorite slate, locality unknown.

Augite porphyry, locality unknown. Mica slate, locality unknown.

Gneiss, Odenwald, Germany.

Two specimens of Scotch granite, localities unknown.

Tincal, or native borax, from East Indies.

Datholite.
Phosphoric acid.

'l'race; had not enough for a second analysis.

Distinct traces.

I obtained so large a precipitate, that I think it must form an important constituent of this mineral.

Present in considerable quantity.

Minute traces, but more abundant in another specimen from Russia.

Enough to give all the usual tests from about $1 \frac{1}{2}$ ounce.

Sufficient from two ounces to give a precipitate with nitrate of silver.

Considerable traces.

Enough for nitrate of silver test.

Considerable traces from two ounces.

Large precipitate from two ounces.

The quantity of phosphoric acid in this substance was very considerable, being equal in one instance to $2 \cdot 13$ per cent. I think most native borax will be found to contain phosphate of soda.

In cne specimen of this mineral from Andreasberg in the Harz, the quan. tity of this acid present was sufficiently large to be estimated. I also detected its presence in a specimen from North America, but in neither case had I enough of the mineral to make another analysis.

It is very curious that such a large quantity of phosphoric acid should in these two cases be associated with boracic acid, which is of a decided volcanic origin. It would be very interesting if the substances occurring with the native boracic acid in Tuscany, and also the other minerals containing boracic acid, were examined for phosphoric acid. The quantitative determination of the phosphoric acid in rocks would, in my opinion, be of little importance, as phosphoric acid, although a constant, is a very variable constituent. This, however, does not apply to the simple minerals, such as olivine, of which it forms very probably a constant constituent.

Dublin, July 17, 1845.

XXVI. Some Observations on the Theory and Application of Electricity. By F. C. Hen RICI *.

1. T $T$ is usually considered, that in consequence of the sup1 posed repulsion exerted between the particles of elec-

* From Poggendorff's Annalen, vol. Ixiv. p. 345. 
tricity of the same kind, the free electricity of any conductor is diffused over its surface and forms a layer of inconsiderable thickness. But, on the one hand, if the electricity were thus forced towards the surface, there must be something which forces, consequently electricity, in the interior of the conductor, and again the repelled particles must exert an equal but opposing action, inasmuch as they cannot escape into any surrounding non-conductor. Thus an equilibrium would be formed between all the electrical particles, which conld only consist in an equality of their mutual pressure. In fact, we only find this condition in aëriform bodies which are confined in any given space (as in porous bodies, which have no attraction for them); moreover, in a solid body heated throughout and surrounded by a bad conductor of heat, we find no trace of a distribution of this heat over the surface.

It is difficult to understand what is the nature of the repulsion supposed to exist between the particles of like electricities. When repulsion occurs between two such particles, both must evidently separate from one another, consequently a fluid consisting of such particles must be expansible, and vice versâ compressible. The mathematical theory of electricity hitherto adopted requires, however, that the electricities should be incompressible fluids. This assumption is however by no means supported by analogy. In ponderable bodies we find a repulsion of the ultimate molecules in gases only, i. e. in remarkably compressible bodies alone; in liquids, properly so called, which are always to a certain extent compressible, we find an attraction of their particles.

The ordinary view of the electrical principle is consequently not capable of explaining the action of electrified bodies at a distance, which is produced as if their free electricity existed only at the surface, and altogether we must consider it merely of use as a means of handling electrical phænomena, and as enabling us to treat the same mathematically.

2. The current from an electrical machine produces galvanic (magnetic, thermal, chemical, \&c.) effects, just as the current from a galvanic series. We can, without difficulty, reduce two currents, one originating in each manner, to exactly the same galvanic action. In this case the electrical particles must have the same quantity of motion in both.

But what a marked difference exists in their tension! and yet, according to the dualistic hypothesis, the separation and reunion of the electricities in the conducting circles of each must take place with equal energy if the above-mentioned conditions are to be fulfilled. Hence it appear's natural that there should exist different kinds of electricity, just as there 


\section{M. Henrici on the Theory and Application of Electricity.}

are different kinds of light, heat and sound. Electrical phænomena would then also be undulatory. We certainly do not readily see how the positive and negative could in this way be accounted for; but such a difficulty cannot justify us in rejecting an explanation in other respects so accordant with reason. Viewed in this light, the increased effect produced by the simultaneous action of several sources of electricity (the voltaic pile, the electrical battery) would be considered as caused in the same way, as by the simultaneous action of many sources of the same kind of light an increased illumination occurs, and in the simultaneous emission of several sounds an increase in the sound conveyed to the ear is produced. The action of the condenser would then be intelligible as converting electricity of less into that of higher tension.

I may be permitted here to draw attention to the facts which electrical phænomena seem to point out, that an essentially different mode of propagation of the electricity occurs in the so-called non-conductors to what takes place in the conductors. Thus it appears that in the former, when they allow the electricity to be diffused to any extent, as the air, its propagation follows the same laws as light and radiant heat ; this is especially pointed out by the late experiments of Petrina, who found that electricity, when propagated in a rectilinear direction in the atmosphere, in passing by a screen (a metallic conducting plate connected with the earth), suffers diffraction, just as rays of light and heat do. Hence any substance would be so much a better or worse conductor than another as it was suitable to either one or the other method of propagation of the electricity; with imperfect conductors both must occur to a certain extent. In other respects the non-conductors of electricity can only be compared within narrow limits with transparent bodies, because in the former the diffusion of the electricity will always be proportionally very limited; the so-called non-conductors of electricity will always be comparatively opake substances when considered electrically. It must also be admitted that they may be compelled by a sufficiently strong electrical power to propagate the electricity by conduction through more or less limited spaces (electric sparks, lightning). It moreover deserves to be noticed, that there is no conduction of heat and light in the same sense as occurs with electricity. We are unacquainted with any diffusion of light within bodies which can be compared to the conduction of electricity, and the diffusion of heat within bodies not only obtains with infinitely less rapidity, but also according to entirely different laws from the conduction of electricity (the same holds good with sound). 
Magnetism exhibits the remarkable property of being retained or absorbed by no other bodies than the few magnetic metals, whilst it is diffused through these comparatively slowly, and in a manner more analogous to heat. Consequently there is no conduction of magnetism at all resembling the conduction of electricity.

If the appearances of light, heat, electricity and magnetism, are phænomena of motion, we must arrive at the conclusion that their exceedingly rapid mode of propagation essentially depends upon motion of an imponderable matter (æther), their slow diffusion being essentially dependent on the movements of the atoms of the bodies. An analogy between magnetism and heat consists in the rays emanating from the former principle being absorbed by the magnetic metals, these thus becoming magnetized, just as bodies become warmed by the absorption of heat; but there is this difference between the two agents, viz. that there are but few bodies capable of absorbing the magnetic rays, whilst there is scarcely one which is not able to absorb those of heat. Consequently nearly all bodies may be regarded as completely pervious to the former rays.

3. If we place two equal-sized plates of copper and zinc upon one another, and after contact separate them, when tested by a delicate electrometer they are both electrical, this occurring only after contact. From this it has been concluded that the dissimilar electricities evolved by the contact of heterogeneous metals neutralize one another at those parts where they are in contact, and that a comparatively small portion of them is set in motion. This retention in very good conductors is unintelligible, if no other forces were exerted: these effects however are evidently those of condensation, as Péclet long since proved by experiment; he showed that all smooth metallic surfaces, when in contact, even withont intervening layers of varnish, act as condensers. In fact, in the present case, the same effect would be evident if the contact of the zinc with the copper plate occurred at a few physical points only (probably when their surfaces are perfectly smooth, and they are lightly placed upon one another, there is no real contact): this agrees with what we observe, that the effect is weaker in proportion as the surfaces in action are less. Thus the experiment by no means justifies the supposition, that the electricities set free by the contact of heterogeneous metals neutralize one another at the place of contact; it is rather natural to suppose that they possess freedom of motion. The consequence of this will be, that from the almost perfect conduction exerted at the place of contact, they will for the greater part combine again, and that a very small portion of them only 


\section{M. Henrici on the Theory and Application of Electricity.}

is diffused over the two conductors lying in contact. This latter portion however may be further carried away by conduction, and it is evident that by contact compensation must ensue for that which is thus carried away (perhaps absorbed by a condenser, or in some other way).

If the conduction be now completed in a manner requiring no further action by contact, an electric current is produced, the power of which must evidently be proportional to the perfection of the whole conduction, because in any given time a certain quantity only of electricity can escape at the point of contact. This quantity of electricity however always amounts to a very small portion only of that originally produced by the contact, as even the best conduction presents a remarkably greater resistance than the point of contact itself.

Thus the internal condition of an open galvanic series * cannot be compared with a charged electrical jar, because in this the electricities conveyed to it from without are artificially separated from one another by an intervening non-conductor and prevented from neutralizing each other. But even here a certain amount of electricity, although it is but small, and proportional to the conducting power of the separating nonconductor, must evidently pass through this to produce neutralization. The discharge of the jar ensues when any better conduction than by means of the non-conductor is presented to the electricities, as for instance, by the air, or still more the earth (which affords in the case of Volta's pile very imperfect conduction). Consequently, an electric jar, if no discharge occurred through the air, would almost entirely part with the electricity communicated to it by rapid discharge through the completed circuit, whilst of the electricity produced by contact a small quantity only can ever be collected and made effective at one time.

From these considerations, it cannot be allowed that the electricity from contact in the experiments we have investigated is capable of being rendered perceptible to the electroscope withuut condensation, because, as we have stated, even between unvarnished and very small metallic surfaces condensatory effects occur. I have succeeded, by the following method of proceeding, in making the electricity of contact unequivocally perceptible without condensation. A strip of silver was fastened to one end of a strip of zinc, the whole was then bent into the form of a $\mathrm{U}$; between the two arms a narrow strip of gold leaf was suspended, so that it could be moved without

- By an open but complete series, as is well known, we understand one in which, if we adopt the contact theory, the series is complete, as expressed by the formula, "Copper-zinc-liquid-copper." 
difficulty to and fro. By carefully moving this gold leaf, it was very easy to place it in such a position that it should be attracted by the strip of zinc only when negatively, and the strip of silver only when positively electrified. As it was electrified by induction, on removing the electrifying bodies (sealing-wax and glass gently excited by friction) the opposite attraction to that produced by the approximation of them took place if an attraction had been thus produced. In my opinion we have here no ambiguily. There is scarcely occasion to remark, that the apparatus was enclosed in a glass vessel. As the normal attraction ensued only when the gold leaf was in a certain position (i. e. exactly midway between the arms of the conpound strip), the small intensity of the electricity of contact is perfectly intelligible. Thus if the gold leaf be approximated to either arm, its free electricity will overpower the weak electricity of contact of this arm, and act upon it by induction, whence attraction between the two must always ensue, as the experiment indicates. I have some hopes of being able to obtain a comparative admeasurement of the intensity of the electrical effects of contact by this method.

4. It is well known that free electricity appears in many chemical processes. Thus the fumes of burning potassium are positive, whilst those of burning phosphorus are negative*. Are we hence to conclude that the atoms of the potash contain positive electricity and those of the phosphoric acid negative electricity in excess? Certainly not. We must merely consider that if the fumes of potassium appear positively electrical, the burning potassium itself must possess negative electricity; the conclusion that the potassium contained preponderance of negative electricity would thus be arrived at. However, the phosphorus, which by combustion must possess positive electricity, as the phosphoric acid appears negative, might be regarded as a body containing positive electricity predominantly. There is no doubt that the electricity which appears in these experiments is first produced in the act of combustion; perhaps it arises from the violent separation of the burnt particles from those still unburnt. Besides, this spontaneously disappears in a very short time, even when it is not purposely annihilated, and the precipitated fumes then indicate no further trace of free electricity; the chemical attraction, therefore, of the potash for the phosphoric acid cannot depend upon the action of free opposite electricities with which their atoms are said to be endowed. Neither can we suppose that any latent electricity exists in them, for such would be unable (if so capable per se) to exert any outward action. If, nevertheless, we were to attribute free electricity to the atoms of bodies, and

* Berzelius, Lehrbuch der Chemie, $5^{\text {te }}$ auf. S. 103. 
to deduce hence their chemical attraction, we must evidently admit the existence of a great difference in the quantity of their peculiar electricities in the various atoms of bodies; the most positive bodies must contain the largest amount of positive, and the negative bodies the largest amount of negative electricity. But how can electro-chemical attraction occur, when two positive or negative bodies act on each other? and yet such bodies combine with each other in the most varied proportions.

Thus it is vain to endeavour to find a physical basis for the electro-chemical views. But there exists between the electrical relations and the general chemical character of a body, a parallelism which cannot be mistaken (as I have already endeavoured to prove elsewhere), and I am still of the opinion that it deserves attentive consideration.

5. The compound galvanic series presents a peculiarity which, in my opinion, deserves to be considered more carefully.

If $n$ represents the cells of like constitution, the electromotive force of which is expressed by $E$, their resistance being $=r$, when united with an homologous metal, and provided with a common conductor whose resistance is $=r$ (previously to which the circuit is not perfectly complete), the intensity of the current (Q) in the latter is

$$
\mathrm{Q}=\frac{n \mathrm{E}}{r+n r^{\prime}} \text {. }
$$

When the elements are arranged in the form of a pile, the intensity of the current $Q^{\prime}$ is

$$
\mathrm{Q}^{\prime}=\frac{n \mathrm{E}}{n r+r^{\prime}}
$$

When $r^{\prime}$ is $=r$, we have $Q=\mathbf{Q}^{\prime}=\frac{n \mathrm{E}}{(n+1) r}$,

and it is then a matter of indifference in what manner the elements are combined as regards the effects. If we now examine the intensity of the current in the elements of the compound series, theory gives the equation

$$
q=\frac{\mathrm{E}}{r+n r^{\prime}}
$$

we thus arrive at the remarkable conclusion, that when $r^{\prime}$ is $=r$, the intensity of the currents in the elementary series is only $\frac{1}{n}$ of that contained in the pile series; in other words, the same mechanical (or other) effect of the conductor closing the circuit, produces under these circumstances in the pile a consumption of matter $n$ times greater than occurs in the compound series formed of the same elements. Thus, were it possible, by mechanical arrangements, to render the resist- 
ance $r^{\prime}$ of the conductor completing the circuit $=r$, the expenditure of these arrangements arising from the galvanic consumption of matter in using 1000 elementary series combined in the manner of the compound series, would amount to $\frac{1}{100}$ th only of that caused by their arrangement in the form of a pile. Consequently, in the former combination, the polarization would also be remarkably slight; we might also thus avoid the use of constant series, and in the selection of a liquid might merely take into consideration, in addition to its conducting power, its chemical relation to the two metals, so as to avoid chemical action between them.

If $n$ be very considerable, so that $r$ in $n r^{\prime}$ may be disregarded, we have

$$
\mathrm{Q}=\frac{\mathrm{E}}{r^{r}} \text { and } q=0 \text {; }
$$

in a very large (strictly considered infinite) number of elementary series; the greatest possible effect of the conductor closing the circuit is thus obtained, considering $r^{\prime}$ as one, without any galvanic consumption of matter, for then no electric current exists in the elementary series. Perhaps we can arrive still nearer the mark, as in a very large number of elementary series, not only the thickness of the smooth metallic plates, between which the liquid is enclosed, but also the thickness of the layers of the liquid is reduced to a minimum : thus the whole apparatus may be confined in a comparatively limited space.

XXVII. Contributions to the Knowledge of Conjugate Compounds. By Dr. H. Kolbe, Chemical Assistant in the University of Marburg*.

THE examination of the products arising from the decom1 position of sulphuret of carbon by means of chlorine has led to the discovery of an interesting series of bodies, which belong, almost without exception, to the class of the so-called conjugate compoundst, and among them the substitution of chlorine by hydrogen is particularly observed, which will probably throw considerable light on the constitution of certain organic combinations. These compounds, produced almost by the direst combination of their elements, have such a strong similarity to those considered to belong to organic chemistry, that it is impossible here to draw a distinct line between organic and inorganic bodies.

I have already $\ddagger$ shown that chlorine and sulphuret of carbon carefully dried are decomposed into a mixture of chloride of sulphur and perchloride of carbon; this takes place not 1845.

* Communicated by the Chemical Society; having been read April 7, † "Corps copulés" of Gerhardt. $\quad \ddagger$ Liebig's Annal. vol. xlv. p. 41 . 